Acta Crystallographica Section F

Structural Biology

and Crystallization

Communications

ISSN 1744-3091

\section{Tatyana N. Safonova, ${ }^{\text {** }}$ Nadezhda N. Mordkovich, ${ }^{a}$ Konstantin M. Polyakov, ${ }^{\text {a,b }}$ Valentin A. Manuvera, ${ }^{c}$ Vladimir P. Veiko ${ }^{\mathbf{a}}$ and Vladimir O. Popov ${ }^{\mathrm{a}}$}

${ }^{a}$ Bach Institute of Biochemistry, Russian Academy of Sciences, 33 Leninskii Avenue, 119071 Moscow, Russian Federation,

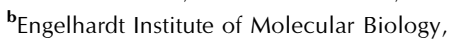
Russian Academy of Sciences, 32 Vavilova Street, 119991 Moscow, Russian Federation, and ${ }^{\mathbf{C}}$ Research Institute for Physico-Chemical Medicine, Federal Medical-Biological Agency, 1a Malaya Pirogovskaya Street, 119435 Moscow, Russian Federation

Correspondence e-mail: tn_safonova@inbi.ras.ru

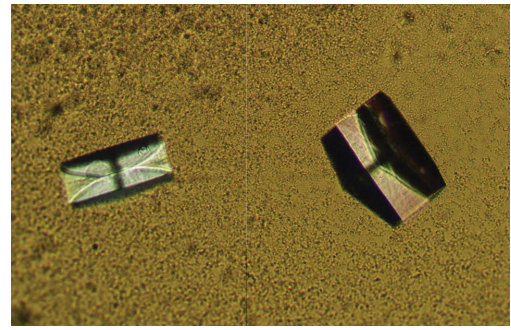

C 2012 International Union of Crystallography All rights reserved

\section{Crystallization of uridine phosphorylase from Shewanella oneidensis MR-1 in the laboratory and under microgravity and preliminary $\mathrm{X}$-ray diffraction analysis}

Uridine phosphorylase (UDP, EC 2.4.2.3), a key enzyme in the pyrimidine salvage pathway, catalyses the reversible phosphorolysis of uridine to uracil and ribose 1-phosphate. The gene expression of UDP from Shewanella oneidensis MR-1 was performed in the recipient strain Escherichia coli. The UDP protein was crystallized on earth (in the free form and in complex with uridine as the substrate) by the hanging-drop vapour-diffusion method at $296 \mathrm{~K}$ and under microgravity conditions (in the free form) aboard the Russian Segment of the International Space Station by the capillary counter-diffusion method. The data sets were collected to a resolution of $1.9 \AA$ from crystals of the free form grown on earth, $1.6 \AA$ from crystals of the complex with uridine and $0.95 \AA$ from crystals of the free form grown under microgravity. All crystals belong to the space group $P 2_{1}$ and have similar unit-cell parameters. The crystal of uridine phosphorylase grown under microgravity diffracted to ultra-high resolution and gave high-quality X-ray diffraction data.

\section{Introduction}

Uridine phosphorylase (UDP, EC 2.4.2.3), a key enzyme in the pyrimidine salvage pathway, catalyses the reversible phosphorolysis of uridine to uracil and ribose 1-phosphate (or 2'-deoxyuridine to 2 -deoxyribose 1-phosphate). UDP is present in most organisms including prokaryotes, yeast and higher organisms. Its level is often elevated in tumours (Krenitsky et al., 1965; Kanzaki et al., 2002). The amino acid sequence of UDP is highly conserved among bacterial and vertebrate UDP (Pugmire \& Ealick, 2002). Upon availability of ribose 1-phosphate, UDP can also catalyse the formation of nucleosides from uracil, as well as from 5-fluorouracil; therefore, it is involved in fluoropyrimidine metabolism and, consequently, the modulation of its enzymatic activity may have an effect on the therapeutic efficacy of these chemotherapeutic agents (Cao et al., 2011). The substrate of UDP, uridine, is essential for RNA synthesis, assembly of biological membranes and regulation of some biological processes.

Uridine phosphorylase from Shewanella oneidensis MR-1 (SoUDP) consists of 252 amino acid residues and has a molecular weight of $27 \mathrm{kDa}$. S. oneidensis MR-1 is a free-living gram-negative $\gamma$-proteobacterium (Alteromonadales order) and is a facultative anaerobe capable of surviving and proliferating in both aerobic and anaerobic conditions. This bacterium is of interest, in particular, due to its application in microbial fuel cells for efficient electricity generation (Lanthier et al., 2008; Lovley, 2008). The activity of SoUDP is 2.5 times higher (Mordkovich et al., 2012) compared with Escherichia coli UDP and is similar to that of UDP from Salmonella typhimurium.

Of the protein structures that have been deposited in the Protein Data Bank, SoUDP has the highest identity with UDP from Vibrio cholerae O1 Biovar El Tor (76\%, PDB code 3o6v, Center for Structural Genomics of Infectious Diseases, unpublished work; $1.70 \AA$ resolution), UDP from S. typhimurium (76\%, PDB code 3ddo, A. A. Lashkov, A. M. Mikhailov \& A. G. Gabdoulkhakov, unpublished work; $1.50 \AA$ resolution), UDP from E. coli (75\%, PDB code 1rxy, Caradoc-Davies et al., 2004; $1.70 \AA$ resolution), and much lower identity with UDP from Streptococcus pyogenes (37\%, PDB code 3qpb, Tran et al., 2011; 1.82 A resolution), bovine UDP (31\%, PDB 


\section{crystallization communications}

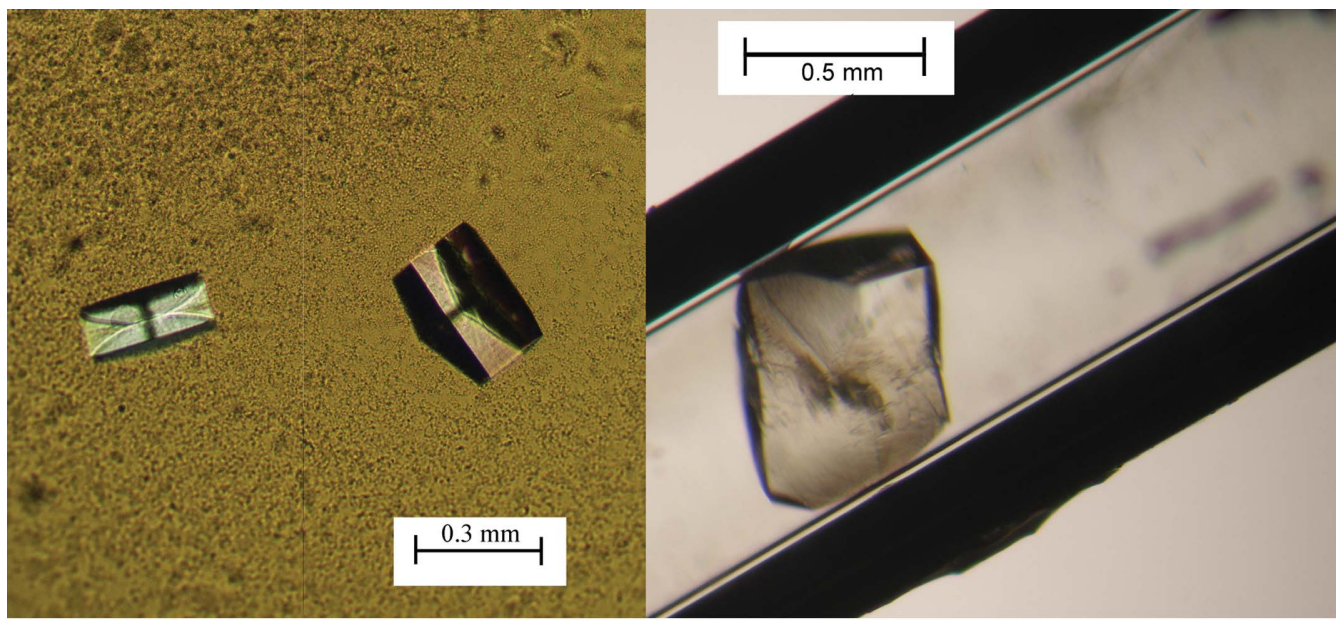

(a)

(b)

Figure 1

Crystals of SoUDP grown $(a)$ in the laboratory and $(b)$ under microgravity conditions and used for X-ray diffraction collection.

code 3ku4, Paul et al., 2010; $2.10 \AA$ A resolution) and human UDP (26\%, PDB code 3euf, Roosild et al., 2009; $1.90 \AA$ resolution). The three-dimensional structure of SoUDP has not been studied as yet.

Here we report the expression, purification, crystallization (in the laboratory on earth and under microgravity), and preliminary X-ray diffraction analysis of SoUDP both in the free form and in complex with uridine.

\section{Experimental procedures}

\subsection{Expression and purification of UDP}

The gene expression of $S$. oneidensis MR-1 udp (GenBank GQ294526) in the recipient strain E. coli was performed as described previously (Mordkovich et al., 2012). Transformants of E. coli $\mathrm{K}-12$ C600 $\Delta m e t-u d p / p S U D P$ were cultured in an incubator shaker at

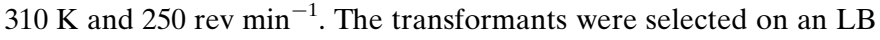
medium containing $50 \mu \mathrm{g} \mathrm{ml}^{-1}$ kanamycin. The biomass of the overnight culture was collected by centrifugation, resuspended in $20 \mathrm{~m} M$ Tris- $\mathrm{HCl}$ pH 8.0 and disrupted with an Ultrasonic Processor ultrasonic disintegrator (Cole Parmer, USA). The cell lysate was centrifuged at $15000 \mathrm{rev} \mathrm{min}^{-1}$ for $30 \mathrm{~min}$. The supernatant was placed on a chromatographic column $(25 \times 1 \mathrm{~cm})$ containing Fractogel TSK DEAE-650M (Merck, Germany). The elution was performed with a linear gradient from 0 to $1 \mathrm{M} \mathrm{NaCl}$ in $20 \mathrm{~m} M$ Tris$\mathrm{HCl} \mathrm{pH} 8.0(200 \mathrm{ml})$ at a flow rate of $1 \mathrm{ml} \mathrm{min}^{-1}$. The collected fractions were analysed using denaturing gel electrophoresis as described by Laemmli (1970). The UDP-containing fraction was transferred into $20 \mathrm{~m} M$ Tris- $\mathrm{HCl}$ buffer $\mathrm{pH} 8.0$ (5 ml) by ultrafiltration using an Amicon YM10 filter (Millipore, USA) and placed on the same chromatographic column. The elution was performed with a linear gradient from 0 to $0.5 \mathrm{M} \mathrm{NaCl}$ in $20 \mathrm{~m} M$ Tris- $\mathrm{HCl} \mathrm{pH} 8.0$ $(250 \mathrm{ml})$. The UDP-containing fraction was transferred into bidistilled water by ultrafiltration. The samples were lyophilized and stored at $253 \mathrm{~K}$.

\subsection{Crystallization}

The initial crystallization experiments for SoUDP in the free form were performed using Crystal Screen, Crystal Screen 2, Crystal Screen Cryo and Index kits from Hampton Research. The screening was performed by the hanging-drop vapour-diffusion method at room temperature. For the crystallization, the lyophilized protein was dissolved in Milli-Q water (the concentration was $20 \mathrm{mg} \mathrm{ml}^{-1}$ ). The solution was centrifuged immediately before crystallization using a

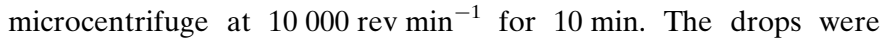
composed of equal volumes $(1 \mu \mathrm{l})$ of reservoir and protein solutions. Drops were equilibrated against $0.5 \mathrm{ml}$ reservoir solution in the wells. The first crystals appeared within 1 week. The best crystals were grown with the use of $0.1 M$ HEPES, $\mathrm{pH} 7.0,5 \%(v / v)$ Tacsimate, $\mathrm{pH}$ $7.0,12 \%(w / v)$ PEG MME 5000 as the reservoir solution. The crystal size was $0.35 \times 0.30 \times 0.40 \mathrm{~mm}$.

Crystals of UDP in complex with the substrate (uridine) were grown by cocrystallization using the hanging-drop vapour-diffusion method at room temperature. Uridine was taken in a 100-fold molar excess with respect to SoUDP. The largest crystals were obtained with the use of $0.75 \mathrm{M}$ ammonium sulfate, $0.075 \mathrm{M}$ Bis-Tris, $\mathrm{pH}$ 5.5, $0.75 \%(w / v)$ PEG $3350,25 \%(v / v)$ glycerol as the reservoir solution. The crystal size was $0.30 \times 0.25 \times 0.30 \mathrm{~mm}$ (Fig. $1 a$ ) .

Since we had a chance to perform a crystal growth experiment under microgravity conditions, we took this opportunity to improve the quality of crystals of UDP in the free form. The conditions for crystallization under microgravity were optimized and converted to the free-interface-diffusion method by performing experiments in glass capillaries (Confocal Science Inc.) $60 \mathrm{~mm}$ in length and $0.3 \mathrm{~mm}$ in inner diameter plugged with silicone tubes filled with agarose gel. The chosen preliminary conditions were used for crystallization under microgravity conditions in glass capillaries with an inner diameter of $0.5 \mathrm{~mm}$ using a JAXA (Japan Aerospace Exploration Agency) crystallization box (JCB) aboard the Russian Segment of the International Space Station (ISS). The filling of capillaries with solutions and the packing of crystallization boxes for launching to the ISS were performed by JAXA researchers according to the protocol developed by the JAXA (Takahashi et al., 2010). Nine different conditions were tested. We succeeded in growing a high-quality crystal using $0.75 M$ ammonium sulfate, $0.1 M$ Bis-Tris, $\mathrm{pH}$ 5.5, $0.75 \%(w / v)$ PEG $3350,25 \%(v / v)$ glycerol. The crystal size was $0.60 \times$ $0.50 \times 0.50 \mathrm{~mm}($ Fig. $1 b)$.

\subsection{X-ray data collection and processing}

For X-ray data collection under cryogenic conditions, the crystal of the free form grown in the laboratory conditions was transferred to a harvesting solution composed of the reservoir solution with the addition of $25 \%$ glycerol for $1 \mathrm{~min}$ and then flash-cooled to $100 \mathrm{~K}$ 
Table 1

Data-collection statistics for uridine phosphorylase from Shewanella oneidensis MR-1.

The data for the last resolution shell are given in parentheses.

\begin{tabular}{llll}
\hline Crystals & $\begin{array}{l}\text { Free form grown } \\
\text { on earth }\end{array}$ & $\begin{array}{l}\text { Complex of UDP } \\
\text { with uridine }\end{array}$ & $\begin{array}{l}\text { Free form grown } \\
\text { under microgravity }\end{array}$ \\
\hline Space group & $P 2_{1}$ & $P 2_{1}$ & $P 2_{1}$ \\
Unit-cell parameters & & & \\
$a(\AA)$ & 90.86 & 91.92 & 91.54 \\
$b(\AA)$ & 94.93 & 96.48 & 95.93 \\
$c(\AA)$ & 90.91 & 91.93 & 91.61 \\
$\beta\left({ }^{\circ}\right)$ & 120.01 & 120.01 & 119.99 \\
Number of hexamers & 2 & 2 & 2 \\
$\quad$ per unit cell, $Z$ & & 100 & 100 \\
Temperature $(\mathrm{K})$ & 100 & $19.5-1.50$ & $30.0-0.95$ \\
Resolution range $(\AA)$ & $50.0-1.90$ & $(1.55-1.50)$ & $(1.0-0.95)$ \\
& $(2.00-1.90)$ & 453428 & 4370315 \\
Measured reflections & 345460 & $(32367)$ & $(543507)$ \\
& $(32035)$ & 205488 & 825784 \\
Unique reflections & 100013 & $(16739)$ & $(114001)$ \\
& $(11928)$ & $92.5(80.5)$ & $94.8(93.4)$ \\
Data completeness $(\%)$ & $93.5(80.1)$ & $2.2(1.9)$ & $5.3(4.8)$ \\
Multiplicity & $3.4(2.7)$ & $10.8(2.1)$ & $12.2(2.6)$ \\
Average $I / \sigma(I)$ & $9.5(2.8)$ & $6.7(42.3)$ & $8.7(70.8)$ \\
$R_{\text {meas }}^{\dagger}$ & $11.8(63.6)$ & 43.39 & 39.86 \\
Solvent content $(\%)$ & 44.10 & 2.19 & 2.06 \\
Matthews coefficient $^{(} \AA^{3}$ Da $\left.{ }^{-1}\right)$ & 2.22 & & \\
\hline & & & \\
\hline & & &
\end{tabular}

$\dagger R_{\text {meas }}=\sum_{h k l}\{N(h k l) /[N(h k l)-1]\}^{1 / 2} \sum_{i}\left|I_{i}(h k l)-\langle I(h k l)\rangle\right| / \sum_{h k l} \sum_{i} I_{i}(h k l)$, where $N$ is the total number of times that a given reflection is measured.

using a Cryostream Plus cooling device (Oxford Cryosystems Ltd, UK). The X-ray data set was collected to a resolution of $1.9 \AA$ using synchrotron radiation on the $\mathrm{K} 4.4$ beamline on the Belok station for protein crystallography of the Sibir'-2 storage ring at the Kurchatov synchrotron radiation source (Moscow) equipped with a Rayonix SX-165 CCD detector at a wavelength of $0.985 \AA$.

Since the crystal of UDP complexed with uridine was grown in the presence of $25 \%(v / v)$ glycerol, it was simply flash-cooled in liquid nitrogen immediately prior to X-ray data collection. The X-ray diffraction data were obtained to a resolution of $1.6 \AA$ on the EMBL Beamline BW7A of the DESY synchrotron (Hamburg) equipped with a MAR165 detector at a wavelength of $0.812 \AA$.

We succeeded in obtaining a high-quality crystal of UDP in the free form under microgravity. Since the crystal was grown in the presence of $25 \%(v / v)$ glycerol, no cryoprotection was required. The X-ray data set was collected at $100 \mathrm{~K}$ to a resolution of $0.95 \AA$ at the SPring- 8 synchrotron radiation facility (Japan) on the BL41XU beamline equipped with an ADSC Quantum-315 CCD detector at a wavelength of $0.800 \AA$.

All diffraction data sets were collected with the $H K L-2000$ suite (Otwinowski \& Minor, 1997) and processed using the XDS program package (Kabsch, 2010) in the space group $P 2_{1}$. The data-collection statistics are summarized in Table 1. Although the unit-cell dimensions are consistent with higher symmetry, we chose the monoclinic space group because the $R_{\text {meas }}$ was significantly low after scaling in this group (for example, $R_{\text {meas }}$ after scaling the X-ray data for SoUDP in complex with uridine at $2.0 \AA$ resolution was 18.2 and $10.7 \%$ in the hexagonal and monoclinic space groups, respectively). All structures were solved by the molecular-replacement method using MOLREP
(Vagin \& Teplyakov, 2010) from the CCP4 suite (Winn et al., 2011) with the structure of E. coli UDP (PDB code 1rxy, Caradoc-Davies et al., 2004) as the starting model. The refinement using REFMAC5 (Murshudov et al., 2011; Winn et al., 2011) is currently in progress.

\section{Results}

We obtained crystals of SoUDP in the free form and in complex with uridine suitable for X-ray diffraction study. The SoUDP has a hexameric structure. The structure solution and first cycles of the structure refinement of SoUDP complexed with the substrate showed that uridine (rather than uracil and ribose) and a sulfate ion (sulfate was present in the reservoir solution) occupying the phosphate binding site are present in the active site of the enzyme. The crystal grown under microgravity aboard the Russian Segment of the ISS was of ultra-high quality. The X-ray diffraction data set from this crystal was collected to a resolution of $0.95 \AA$. The atomic resolution can help in a better understanding of enzymatic properties and fine structural details for future drug design.

We thank the staff of the SPring- 8 synchrotron radiation facility (Japan) for help during synchrotron data collection. This work was supported by the Ministry of Education and Science of the Russian Federation (Federal Target Programme 'Research and Development in Priority Fields of Science and Technology Complex of Russia for 2007-2012'; Federal Contract No. 16.512.11.2174).

\section{References}

Cao, D., Ziemba, A., McCabe, J., Yan, R., Wan, L., Kim, B., Gach, M., Flynn, S. \& Pizzorno, G. (2011). Mol. Cancer Ther. 10, 2330-2339.

Caradoc-Davies, T. T., Cutfield, S. M., Lamont, I. L. \& Cutfield, J. F. (2004). J. Mol. Biol. 337, 337-354.

Kabsch, W. (2010). Acta Cryst. D66, 125-132.

Kanzaki, A., Takebayashi, Y., Bando, H., Eliason, J. F., Watanabe Si, S., Miyashita, H., Fukumoto, M., Toi, M. \& Uchida, T. (2002). Int. J. Cancer, 97, 631-635.

Krenitsky, T. A., Mellors, J. W. \& Barclay, R. K. (1965). J. Biol. Chem. 240, 1281-1286.

Laemmli, U. K. (1970). Nature (London), 227, 680-685.

Lanthier, M., Gregory, K. B. \& Lovley, D. R. (2008). FEMS Microbiol. Lett. 278, 29-35.

Lovley, D. R. (2008). Curr. Opinion Biotechnol. 19, 1-8.

Mordkovich, N. N., Manuvera, V. A., Veiko, V. P. \& Debabov, V. G. (2012). Biotekhnologiia (Moscow), 1, 21-30.

Murshudov, G. N., Skubák, P., Lebedev, A. A., Pannu, N. S., Steiner, R. A., Nicholls, R. A., Winn, M. D., Long, F. \& Vagin, A. A. (2011). Acta Cryst. D67, 355-367.

Otwinowski, Z. \& Minor, W. (1997). Methods Enzymol. 276, 307-326.

Paul, D., O'Leary, S. E., Rajashankar, K., Bu, W., Toms, A., Settembre, E. C., Sanders, J. M., Begley, T. P. \& Ealick, S. E. (2010). Biochemistry, 49, 34993509.

Pugmire, M. J. \& Ealick, S. E. (2002). Biochem. J. 361, 1-25.

Roosild, T. P., Castronovo, S., Fabbiani, M. \& Pizzorno, G. (2009). BMC Struct. Biol. 9, 14

Takahashi, S., Tsurumura, T., Aritake, K., Furubayashi, N., Sato, M., Yamanaka, M., Hirota, E., Sano, S., Kobayashi, T., Tanaka, T., Inaka, K., Tanaka, H. \& Urade, Y. (2010). Acta Cryst. F66, 846-850.

Tran, T. H., Christoffersen, S., Allan, P. W., Parker, W. B., Piskur, J., Serra, I., Terreni, M. \& Ealick, S. E. (2011). Biochemistry, 50, 6549-6558.

Vagin, A. \& Teplyakov, A. (2010). Acta Cryst. D66, 22-25.

Winn, M. D. et al. (2011). Acta Cryst. D67, 235-242. 